\title{
Optimal assembly strategies of transcriptome related to ploidies of eukaryotic organisms
}

\author{
Bin $\mathrm{He}^{\dagger}$, Shirong Zhao ${ }^{\dagger}$ Y Yuehong Chen, Qinghua Cao, Changhe Wei, Xiaojie Cheng and Yizheng Zhang*
}

\begin{abstract}
Background: Several de novo transcriptome assemblers have been developed recently to assemble the short reads generated from the next-generation sequencing platforms and different strategies were employed for assembling transcriptomes of various eukaryotes without genome sequences. Though there are some comparisons among these de novo assembly tools for assembling transcriptomes of different eukaryotic organisms, there is no report about the relationship between assembly strategies and ploidies of the organisms.

Results: When we de novo assembled transcriptomes of sweet potato (hexaploid), Trametes gallica (a diploid fungus), Oryza meyeriana (a diploid wild rice), five assemblers, including Edena, Oases, Soaptrans, IDBA-tran and Trinity, were used in different strategies (Single-Assembler Single-Parameter, SASP; Single-Assembler Multiple-Parameters, SAMP; Combined De novo Transcriptome Assembly, CDTA, that is multiple assembler multiple parameter). It was found that CDTA strategy has the best performance compared with other two strategies for assembling transcriptome of the hexaploid sweet potato, whereas SAMP strategy with assembler Oases is better than other strategies for assembling transcriptomes of diploid fungus and the wild rice transcriptomes.

Conclusion: Based on the results from ours and others, it is suggested that CDTA strategy is better used for transcriptome assembly of polyploidy organisms and SAMP strategy of Oases is outperformed for those diploid organisms without genome sequences.
\end{abstract}

Keywords: Transcriptome assembly, RNA-Seq, Diploid, Polyploidy, Sweet potato, Oryza meyeriana, Trametes gallica

\section{Background}

Transcriptome sequencing projects for non-model organisms have revolutionized the field of biology and medical research and impressively enlarged the realm of transcriptomic analyses, because they cost less and are more computationally tractable than full genome sequencing projects [1]. For instance, these new technologies have been efficiently employed in the discovery of new genes [2], the development of new tissue specific or cancer biomarkers [3], the isolation of fast-evolving genes [4], the detection of new alternative splice variants [5], allelespecific gene expression [6], SNP discovery in genes, or epigenetic gene regulation [7].

However, these new sequencing technologies also brought tremendous challenges to traditional de novo

\footnotetext{
* Correspondence: yizzhang@scu.edu.cn

${ }^{\dagger}$ Equal contributors

Key Laboratory of Bio-resources and Eco-environment, Ministry of Education, Sichuan Key Laboratory of Molecular Biology and Biotechnology, College of Life Sciences, Sichuan University, 610064 Chengdu, China
}

assembly tools designed for Sanger sequencing, as they are incapable of handling the millions to billions of short reads (35-400 bp each) generated by next-generation sequencing platforms $[1,8]$. Meanwhile, the size and quality of the assembled transcriptome seriously affect the subsequent studies. Therefore, several novel de novo assembly tools and strategies have been developed, such as ABySS [9], SSAKE [10], Edena [11], Oases [12], Soaptrans [13], Soapdenovo [14], IDBA-tran [15], Trinity [16] and combined de novo transcriptome assembly strategy (CDTA) $[17,18]$. However, most assemblers and strategies have been employed for assembling transcriptomes of the same species or different organisms with the same ploidies. For instance, Garg et al. compared the performance of Oases, Abyss, Soapdenovo and commercially available CLC Genomics workbench in chickpea (Cicer arietinum L. genotype ICC4958) [19]. They obtained the conclusion that the assembly of short-read data set obtained by Oases was found better than others. Zhang et al. employed various assemblers and reported the comparison of de novo 
assembly software tools among Swinepox virus (Swinepox), Escherichia coli str. K-12 substr (bacterium), Saccharomyces cerevisiae (a diploid yeast) and Caenorhabditis elegans (a diploid nematode) [20]. Their conclusion indicated that overlap-layout-consensus (OLC) assemblers are well-suited for very short reads and longer reads of small genomes respectively. For large datasets of more than hundred millions of short reads, De Bruijn graph-based assemblers would be more appropriate. Zhang et al. compared five assemblers (MIRA, Newbler, SOAPdenovo, SOAPdenovo-trans [SOAPtrans], Trinity) to determine the optimal transcriptome sequencing approach in Geranium maderense and Pelargonium $x$ hortorum [21]. They found that Trinity or SOAPtrans generate high-quality de novo transcriptomes with broad coverage. Apparently, different researchers made their own conclusions. In addition, the previous reports on comparsion of assemblers demonstrated the influence of the length of reads, the type of reads and sequencing platform, while ignored the relationship between the assembly strategies and the ploidies of organisms investigated [22-24].

Actually, all transcriptome assembly strategies could be summarized as three types. It was well-known that some assemblers are used in default parameter, such as Trinity, or in optimized parameter, such as CLC genomics workbench. This strategy is called Single-Assembler Single-Parameter (SASP) described below in this paper. It has been known that Trinity is the best assembler in SASP strategy. For other assemblers, different parameters could be chosen to assembly transcriptomes. This strategy is called Single-Assembler Multiple-Parameter (SAMP). In this strategy, the data assembled from different parameters were merged and assembled with CAP3 [17]. In the third strategy, called Combined De novo Transcriptome Assembly (CDTA), the final transcriptome is obtained from emerging data assembled from different parameters of various assemblers (could be also called Multiple-Assemblers Multiple-Parameters, MSMP). This strategy has been used for the transcriptome assembly of sweet potato (hexaploid) [25]. In our previous study, we found that the CDTA strategy was the best one to assembly the sweet potato transcriptome. However, when the same strategy was applied for the transcriptome assembly of a diploid fungus, Trametes gallica, we found that the data were not better than those assembled from SAMP of Oases. Afterwards, very similar results were obtained from the transcriptome assembly of the diploid wild rice, Oryza meyeriana. Intuitively, we suspect that the ploidies of species should have a significant impact on choosing de novo assemblers and strategies.

Accordingly, in this study, we systematically compared the performance of SASP, SAMP and CDTA strategies in assembling transcriptomes of sweet potato, wild rice and fungus. Based on the results from ours and others, we provided guidelines for the selection of optimal assembly strategy for various eukaryotic organisms with different ploidies and useful information for improving current assemblers and developing new high-performance assemblers.

\section{Results}

\section{Sequencing of samples}

We filtered the sequence data for low-quality reads at high stringency (reads with more than $20 \%$ of bases with Phred quality score of $\leq 10$ ), reads with unknown nucleotides larger than $5 \%$ and reads containing primer/adaptor sequence. From Trametes gallica, we obtained a total of $13,274,462$ paired-end reads with $90 \mathrm{nt}$ in length $(66,372,31$ from each end), encompassing about $2 \mathrm{~GB}$ of sequence data in fastq format. From Oryza meyeriana, we obtained a total of 162,133,290 paired-end reads with $90 \mathrm{nt}$ in length (81,066,645 from each end), encompassing about $10 \mathrm{~GB}$ of sequence data in fastq format. From Ipomoea batatas, we obtained a total of 48,716,884 paired-end reads with $100 \mathrm{nt}$ in length (24,358,442 from each end), encompassing about $4 \mathrm{~GB}$ of sequence data in fastq format (Table 1).

\section{Preliminary evaluation of different assembly strategies in individual species}

The de novo assembly of transcriptome was carried out with various assemblers and assembly strategies. We made a preliminary assessment for various assemblers at different k-mer lengths and various assembly strategies in each species from N50 value, number of longer than 1000 bp assembled contigs and average contig size.

In Trametes gallica, when using SASP strategy at different k-mer lengths, we found the best assembly to be the Oases program for $k=21$, as it resulted in the highest N50 length of $1529 \mathrm{bp}$, the most contigs larger than 1000 bp of 8627 and the largest average contig length of $881 \mathrm{bp}$. While using SAMP and CDTA strategy, the results presented that SAMP of Oases has the optimum performance with N50 length of 1624, the most contigs larger than $1000 \mathrm{bp}$ of 10024 and the largest average contig length of $908 \mathrm{bp}$. In addition, CDTA strategy was also well-behaved, just next to SAMP of Oases (Additional file 1: Table S1).

In sweet potato, the IDBA-tran program for $\mathrm{k}=45$ generates the best results in SASP strategy with the highest

Table 1 Summary of data generated for sequencing of samples

\begin{tabular}{lllll}
\hline Species & No. of reads & $\begin{array}{l}\text { No. of } \\
\text { nucleotides } \\
\text { (nt) }\end{array}$ & $\begin{array}{l}\text { Length of } \\
\text { reads (nt) }\end{array}$ & $\begin{array}{l}\text { Type of } \\
\text { reads }\end{array}$ \\
\hline Trametes gallica & $13,274,462$ & $1,194,704,580$ & 90 & Paired-end \\
Oryza meyeriana & $162,133,290$ & $14,591,996,100$ & 90 & Paired-end \\
Sweet patato & $48,716,884$ & $3,653,766,300$ & 100 & Paired-end \\
\hline
\end{tabular}


N50 length of 1194, the most contigs larger than $1000 \mathrm{bp}$ of 20,609 and the largest average contig length of $967 \mathrm{bp}$ (Additional file 2: Table S2). Both CDTA and SAMP of Oases and IDBA-tran displayed a good performance.

In Oryza meyeriana, the Oases program for $\mathrm{k}=27$ and SAMP of Oases were seem to give the best in SASP and SAMP strategy (Additional file 3: Table S3). CDTA strategy also generated ideal results with N50 length of 1,756, contigs larger than 1000 bp of 76,871 and average contig length of $1,132 \mathrm{bp}$.

\section{Performance evaluation by size distribution among various species}

To determine the relation of assemble strategies and species, the information of size distribution of assembled contigs was compared among three species, including N50 value, number of longer than 1000 bp assembled contigs and average contig size.

The preliminary assessment of these de novo assemble strategies showed that evaluation results of SAMP strategy are more advanced than SASP so that we only chose the databases from SAMP strategy and CDTA strategy for further evaluation. The comparison of N50 value showed that SAMP of Oases has a better performance than other strategies in Trametes gallica and Oryza meyeriana, diploid species, while in sweet potato, hexaploid species, CTDA has better N50 value (Figure 1a). The evaluation results from the average contig size and the number of contigs longer than $1000 \mathrm{bp}$ displayed that the SAMP strategy of Oases was better than the CDTA and other strategies for assembling the transcriptomes of two diploid organisms (Figure 1b) and the CDTA strategy was better than the SAMP for sweet potato transcriptome assembly (Figure 1c).

\section{Performance evaluation by accuracy and completeness among various species}

Another optimality criterion for a novel de novo assembled transcriptome is how well it recapitulates previously determined sequences for the target species, and how well it represents sequences from related organisms. The best assembler will return contigs that match previous data well, and will deliver a high coverage of the conserved proteome of related taxa. Through sequence homology search with well-annotated and identified genes in them and phylogenetically related species, we evaluated the accuracy of different de novo assemble strategies. Because of the advanced performance of SAMP strategy, we only evaluated the performance of SAMP strategy of multiple k-mer assemblers. The numbers on $100 \%$ and $80 \%$ of the length coverage of top database hits were counted. The results showed that Oases also has a better performance than other strategies in Trametes gallica and Oryza meyeriana, although it is not very obvious compared with Trinity and CDTA, while in sweet potato, CTDA strategy performed better than other de novo assemblers and strategies (Figure 2). From the accuracy evaluation, CDTA and Oases are more excellent than other de novo assemble strategies, especially compared with Soaptrans no matter in Trametes gallica and Oryza meyeriana or in sweet potato.

In addition to the statistics of accuracy, completeness was also considered to evaluate the quality of the assemblies. As shown in Figure 3, the value of completeness with the SAMP strategy of Oases is higher than any of other de novo assemblers and strategies in Trametes gallica and Oryza meyeriana, whereas the contigs provided by the CTDA strategy have the highest value in sweet potato.

\section{Performance evaluation by long ORF numbers among various species}

Since mRNA was sequenced in RNA-seq and most of mRNA encodes full-length protein, the optimal assembler should produce a large number of long ORFs. To determine the performance of each assembly strategy on long ORF numbers, we count the number of size $900 \mathrm{bp}$ and $1200 \mathrm{bp}$ or longer ORFs. The difference of number of size $900 \mathrm{bp}$ and $1200 \mathrm{bp}$ or longer ORFs is not remarkable and reach a same conclusion. In Trametes gallica and Oryza meyeriana, SAMP of Oases produced long ORFs at most and exceeded much more than SAMP of Edena and Soaptrans (Figure 4). Meanwhile, CDTA strategy has the best performance in sweet potato and more than twice as SAMP of Oases, even of IDBA-tran, which produced the most long ORFs in SAMP strategy.

\section{Discussion}

With the recent introduction of transcriptome sequencing projects, de novo assemblers developed rapidly as well and applied on many species, mainly referring to diploid species and few referring to tetraploid and hexaploid (Table 2). From the previous researches, there was no report particularly interesting in the relationship between ploidies and assembling quality with different de novo assemblers and strategies. In this study, different datasets of Oryza meyeriana, sweet potato and Trametes gallica were generated to address this issue. Our results consistently suggest that the ploidies of species should have a significant impact on the transcriptome quality from different de novo assemblers and strategies. During the de novo assembly of diploid species, the SAMP strategy of Oases performs the best when comparing with other strategies. Though CDTA strategy gave a similar result with the SAMP strategy of Oases, it spent much more time than the SAMP strategy of Oases (dates not show). While during the de novo assembly of hexaploid 


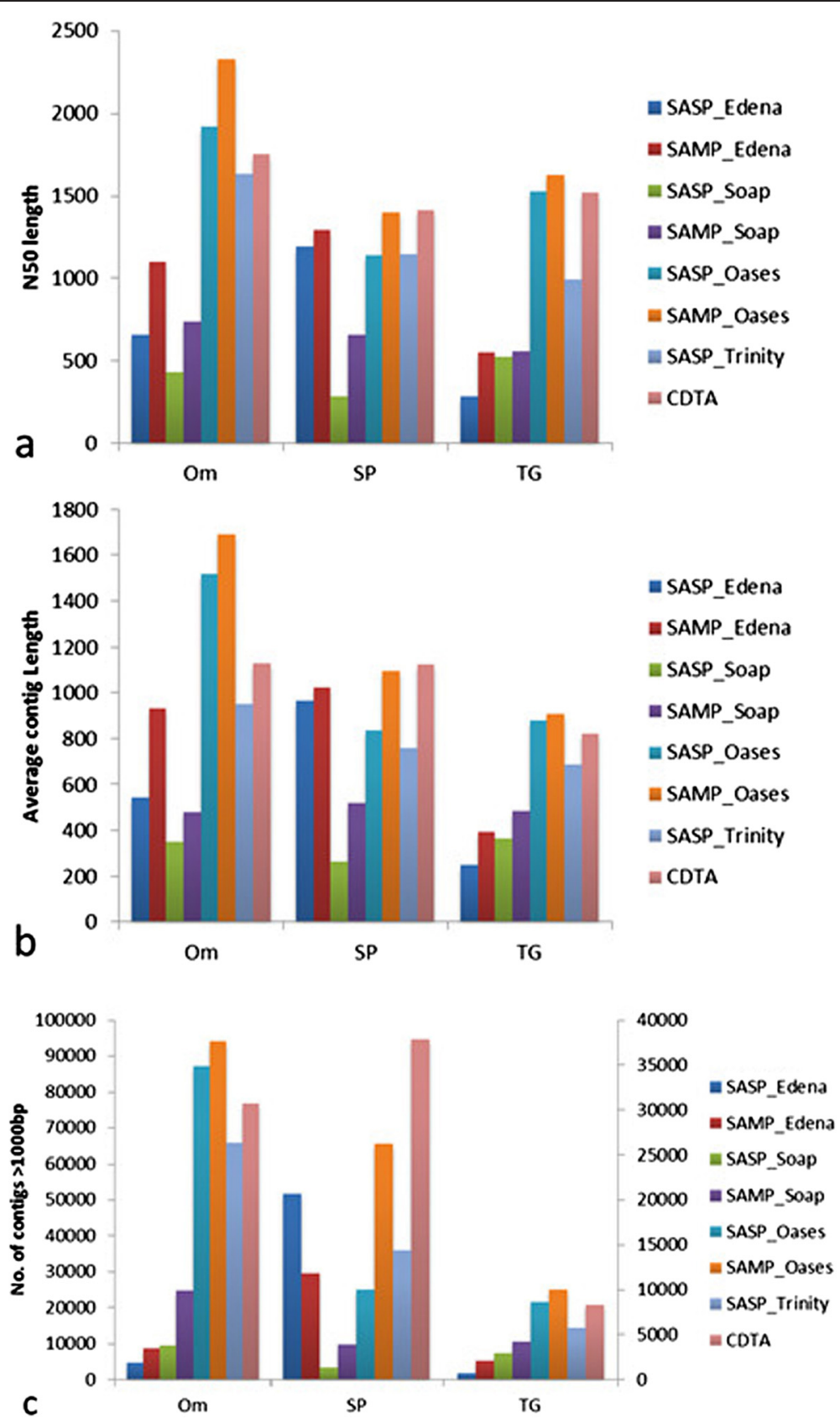

Figure 1 Comparison of size distribution using various de novo assembly tools and strategies in Trametes gallica, sweet potato and Oryza meyeriana. (a) Comparison of N50 length. (b) Average contig length. (c) Number of contigs $>1000$ bp, data of Oryza meyeriana are based on the left $Y$ axes and data of Trametes gallica and sweet potato are based on the right $Y$ axes. Note: Edena assembler in sweet potato actually corresponds to IDBA-tran assembler.

species, the CDTA strategy shows an obvious advantage than other strategies.

To further verify our assembly strategies, we tests our pipelines on other organisms, Zea mays (diploid, SRR 925467) and Triticum turgidum (Tetraploid, SRR863 394). 290 and 200 identified mRNA sequences from Zea mays and Triticum turgidum, respectively, were chosen as reference sequences to evaluate the performance of each assembly strategy. The results showed that our pipelines also applied to other organisms, including diploid and polyploidy species. In Zea mays, SAMP of Oases exhibited superiority on N50 value, numbers of contigs longer than $1000 \mathrm{bp}$ and average contig size, especially compared with soap-trans and Trinity (Additional file 4: Table S4). When blasted to the reference genes, Trinity, SAMP of Oases and CDTA strategy all showed a good 


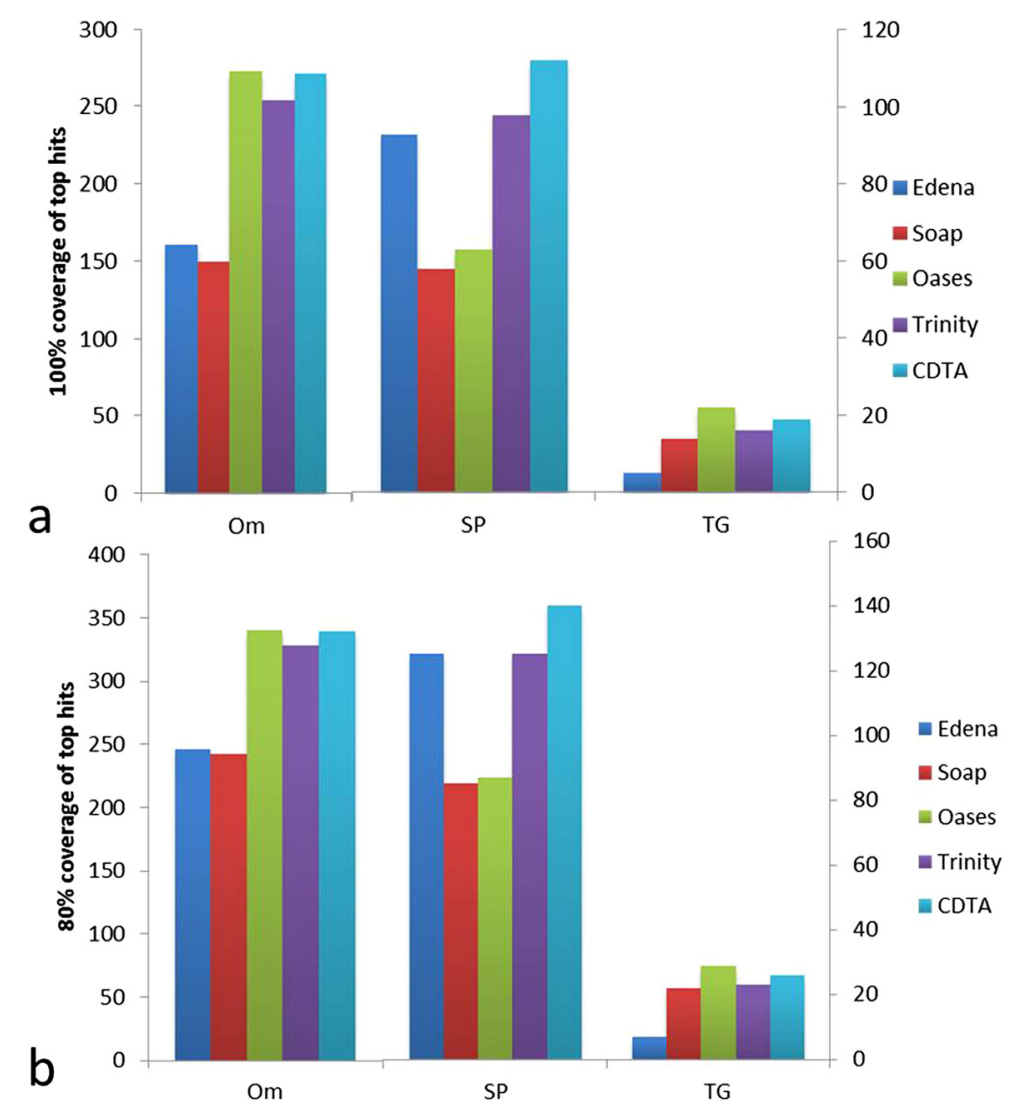

Figure 2 Comparsion of the length coverage of top database hits using various de novo assembly tools and strategies in Trametes gallica, sweet potato and Oryza meyeriana. (a) The numbers on 100\% of the length coverage of top database hits. (b) The numbers on $80 \%$ of the length coverage of top database hits. Data of Oryza meyeriana are based on the left $Y$ axes and data of Trametes gallica and sweet potato are based on the right $Y$ axes. Note: Edena assembler in sweet potato actually corresponds to IDBA-tran assembler.

performance, 144 reference genes were found to be $100 \%$ of the length coverage of top database hits (Additional file 5: Figure S1). The results from the numbers of contigs with $80 \%$ of the length coverage of top database hits were displayed that SAMP of Oases gave better

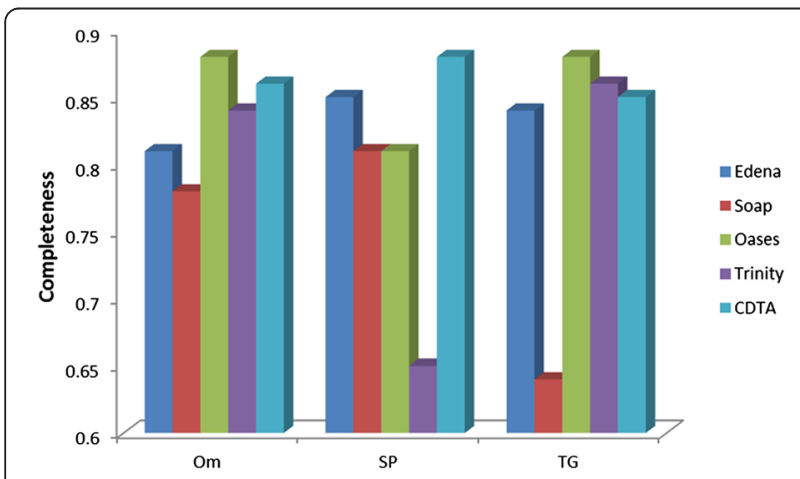

Figure 3 Comparsion of completeness using various de novo assembly tools and strategies in Trametes gallica, sweet potato and Oryza meyeriana. Note: Edena assembler in sweet potato actually corresponds to IDBA-tran assembler. performance compared with other strategies. In addition, the predicted long ORF numbers and completeness confirmed our speculation as well (Additional file 6: Figure S2). While in Triticum turgidum, although the results of CDTA strategy on completeness and numbers of contigs with $100 \%$ and $80 \%$ of the length coverage of top database hits were similar with that of SAMP of Oases, the performance of CDTA on the length statistics and predicted long ORF numbers were more advanced than other strategies (Additional file 7: Table S5). These results were coincidence with our speculation.

There are many researches displaying very similar results with ours. Transcriptome assembly on Chickpea, diploid $(2 \mathrm{n}=2 \mathrm{x}=16)$ plant, showed that Oases performs the best comparing with the performance of Abyss, Soapdenovo and commercially available CLC Genomics workbench [19]. Research on optimizing de novo assembly of short-read RNA-seq data in Ricinus communis showed that SAMP strategy of Oases produced the highest gene coverage among popular assembly packages [31]. In previous transcriptome assembly of sweet potato, CDTA could be a good choice for this hexaploid species, 


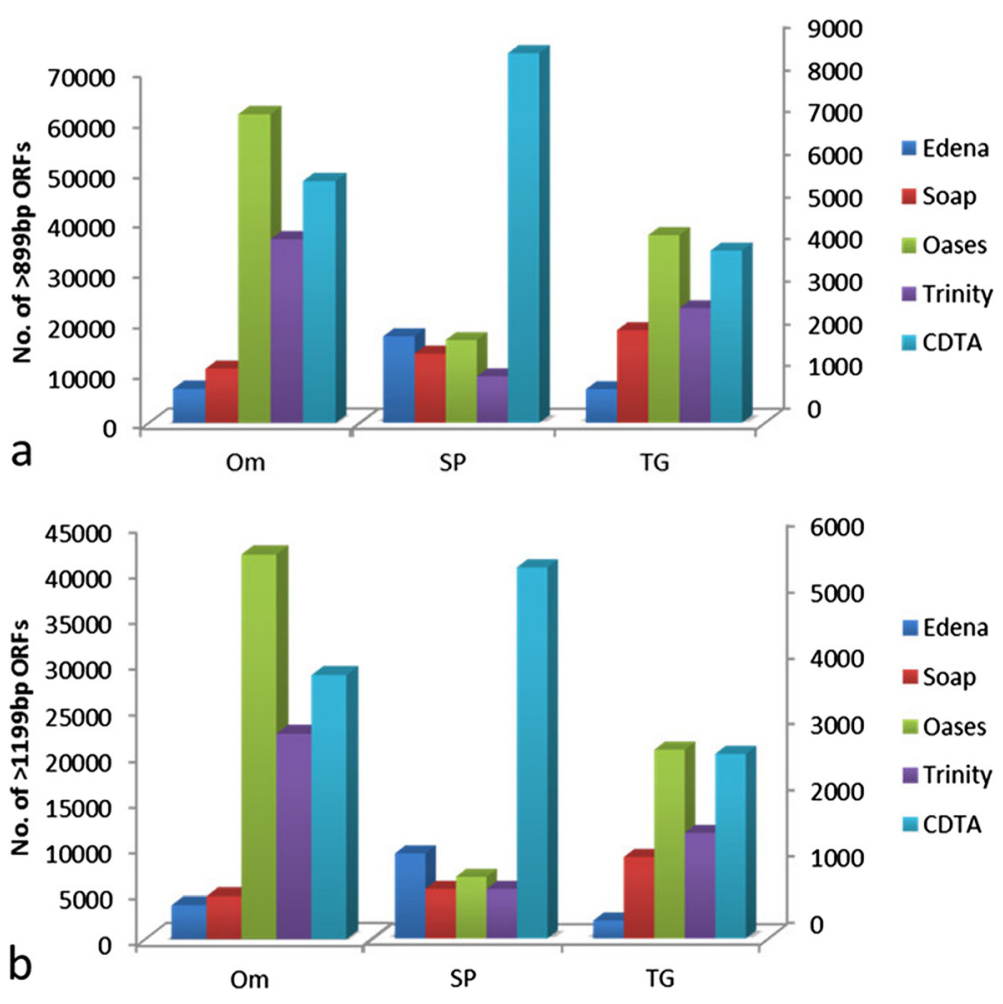

Figure 4 Comparsion of number of long ORFs using various de novo assembly tools and strategies in Trametes gallica, sweet potato and Oryza meyeriana. (a) Number of size 900 bp or longer ORFs. (b) Number of size 1200 bp or longer ORFs. Data of Oryza meyeriana are based on the left $Y$ axes and data of Trametes gallica and sweet potato are based on the right $Y$ axes. Note: Edena assembler in sweet potato actually corresponds to IDBA-tran assembler.

compared with other four assemblers [25]. Furthermore, the research on Nicotiana benthamiana, an allo-tetraploid plant, showed that CDTA strategy has a better performance than other strategies [18].

The assembly of the transcriptome of a polyploid species poses additional problems that are not encountered in diploid species. The studies of hexaploid wheat transcriptomes highlight the difficulties of assembling closely related homoeologs in a polyploid species [32]. Schreiber et al. (2012) [33] observed that most homoeologs were

Table 2 Partial application of various assemblers in different ploidies species

\begin{tabular}{llll}
\hline Species & Ploidies & Assemblers & References \\
\hline Chickpea & Diploid & Oases & {$[19]$} \\
Tea plant & Diploid & Soapdenovo & {$[26]$} \\
Carrot & Diploid & CTDA & {$[17]$} \\
Camelina sativa & Diploid & Trinity & {$[27]$} \\
Pasta wheat & Tetraploid & CDTA & {$[28]$} \\
Nicotiana benthamiana & Tetraploid & CDTA & {$[18]$} \\
Common wheat & Hexaploid & Trinity & {$[29]$} \\
Sweetpotato & Hexaploid & Soapdenovo & {$[30]$} \\
Sweetpotato & Hexaploid & CTDA & {$[25]$} \\
\hline
\end{tabular}

collapsed into chimeric contigs when hexaploid wheat transcriptomes were assembled using either Velvet/Oases (60\% chimeric sequences) or Trinity (50\% chimeric sequences) [33]. Therefore, when mapping reads to the contigs it is important to adjust the number of mismatches to tolerate the average differences generated by genome divergences [32]. In our case, we added additional layers of merging through softwares such as CAP3 with up to 2 nucleotide differences in $40 \mathrm{bp}$ average reads in the first layer. In addition, given assembler-specific optimal parameters, different assemblers can be more efficient at reconstructing different sets of sequences [18]. Therefore, it may be the reason why CDTA strategy has a better performance in the de novo assembly of a polyploid species than other strategies but not the SAMP strategy of Oases.

It is undeniable that there exists discrepancy on depths of coverage among three species we chose. However, all of depths of coverage ensures enough genome coverage and control the sequencing error rate because it exceeds the threshold that would affect the transcriptome research [34]. Therefore, in short, we recommended SAMP strategy of Oases to assembly transcriptome of diploid species. Although CDTA strategy has a good performance on three aspects we evaluated above, Oases costs a lot less time than CDTA strategy. While assembling transcriptome of 
hexaploid and tetraploid species, we recommended CDTA strategy. This conclusion will be vital to those working with transcriptomic data, and will ultimately allow researchers to produce de novo assemble high-performance tools for different ploidies of species without genome sequences.

\section{Conclusion}

In this study, diploid species, Oryza meyeriana and Trametes gallica, and hexaploid species, sweet potato, were sent to transcriptome sequencing to identify the relationship between ploidies and assembling quality with different de novo assemblers. We evaluated the performance of each assembly strategy from the size distribution of assembled contigs, the accuracy and the precision and predicted long Open Reading Frame (ORF) numbers. The performance of evaluation have shown that CDTA strategy is better used for transcriptome assembly of polyploidy organisms and SAMP strategy of Oases is outperformed for those diploid organisms. Therefore, this study will be vital to those working with transcriptomic data, and will ultimately allow researchers to produce de novo assemble high-performance tools for different ploidies of species without genome sequences.

\section{Methods}

\section{Samples and RNA extraction}

The fungal strain of Trametes gallica used in this study were activated on potato dextrose agar (PDA) plate and then inoculated cultured statically in 27 kinds of liquid media and cultured statically at $28^{\circ} \mathrm{C}$ about $10 \mathrm{~d}$. Sweet potato [I. Batatas (L.) Lam. cv. Xushu 18] was grown under normal conditions in Chengdu, Sichuan Province of China [25]. Samples of leaves were collected after planting. Oryza meyeriana was planted at natural temperature and light in Yuanjiang, Yunnan Province of China. Samples of roots, stems and leaves were collected. Trametes gallica and Oryza meyeriana were diploid species, whereas sweet potato was hexaploid species, designated as TG, Om and SP, respectively. Each samples was snapfrozen immediately in nitrogen and stored at $-80^{\circ} \mathrm{C}$ until further processing.

Total RNAs were extracted from each sample by using the Trizol Reagent (Invitrogen, USA), and treated with DNase I (Fermentas, USA) according to the manufacturer's instructions. RNA concentrations were measured with Qubit fluorometer (Invitrogen, USA).

\section{Library construction and Illumina sequencing}

Beads with oligo(dT) were used to purify poly(A) mRNA from total RNA. Then, the mRNA was fragmented using a RNA fragmentation kit (Ambion). First strand cDNAs were synthesized using Oligo(dT) primer, then second strand cDNAs were synthesized using RNase $\mathrm{H}$ and
DNA polymerase I. Double stranded cDNAs were random fragmented using Nebulizer, then repaired and added an adenine base to the $3^{\prime}$ end. Then the pairedend cDNA library was prepared with an insert size of $200 \mathrm{bp}$ and submitted to Illumina GA II platform for sequencing at Beijing Genomics Institute (BGI)-Shenzhen, Shenzhen, China (http://www.genomics.cn).

\section{Read pre-processing}

To ensure that the raw data looks good and there are no problems or biases, pair-end raw reads were performed some simple quality control as implemented by fastqc version 0.10 .1 [35], including per base sequence quality, per sequence quality scores, per base sequence content, per base GC content and so on. The reads with low scores of less than 20 at $3^{\prime}$ end were filtered out.

\section{Assembly and strategies}

In order to obtain optimal assembling results, three strategies, SASP, SAMP and CTDA, were employed by using four commonly de novo assemblers to assemble transcriptomes of above three organisms.

Trinity_release_20131110 (http://trinityrnaseq.github.io/) [16], which used in default parameter, kmer $=25$, was used in SASP strategy and its Command-line parameters were “-seqType fq -left Reads_1.fq -right Reads_2.fq -CPU 20".

Three common multiple k-mer de novo assemblers, including Edena V3.130110 (www.genomic.ch/edena.php) [11], Oases V0.2.8 (www.ebi.ac.uk/ zerbino/oases/) [12], Soaptrans Release 1.03 (http://sourceforge.net/projects/ soapdenovotrans/files/SOAPdenovo-Trans/) [13] and IDBA-tran V1.1.1 (http://www.cs.hku.hk/ alse/idba tran) [15], were respectively used in SAMP strategy. Edena was first run by using a set of $\mathrm{k}$-mer values and the contig databases obtained from k-mer 40, 45, 50, 55 and 60 were then merged into one contig database with CAP3 [36]. The same strategy as Edena were employed to make assembly by using Soaptrans, Oases and IDBA-tran. Oases was run by using k-mer values of 21, 23, 25, 27 and 29, and Soaptrans was run by the same strategy using k-mer values including 37, 41, 45, 49 and 53, while IDBA-tran was run by using k-mer values of 41, 43, 45, 47 and 49 .

In combined de novo transcriptome assembly strategy (CTDA), all contig pools from four assemblers described above were merged and reassembled with CAP3. The detailed pipelines of SASP, SAMP and CDTA is shown in Figure 5. All the work of assemblies was run on a 64-bit Linux system (Ubuntu 10.10) with 256G physical memory.

\section{Performance evaluation}

To further evaluate the performance of each assembly strategy, the size distribution of assembled contigs, the 


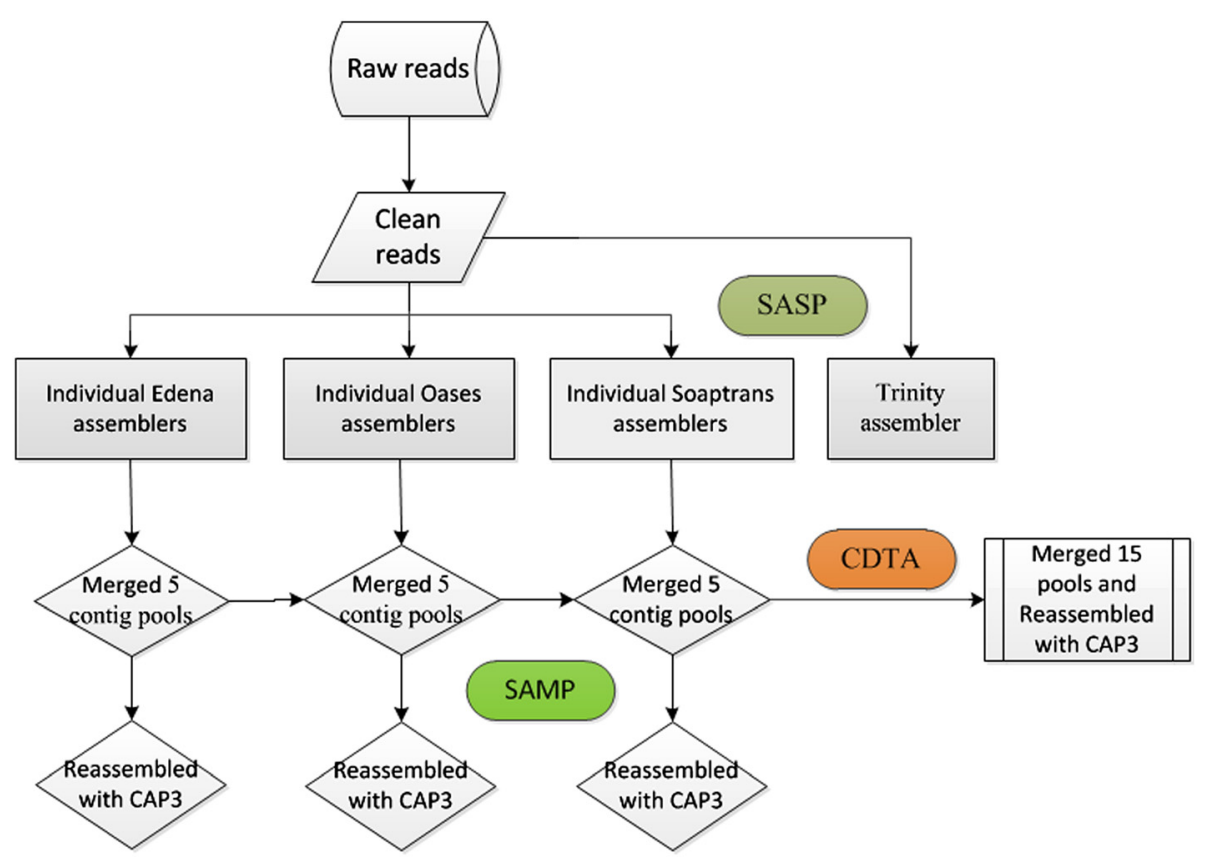

Figure 5 The detailed pipeline of SASP, SAMP and CDTA strategy.

accuracy and the precision and predicted long Open Reading Frame (ORF) numbers, were evaluated.

In the first statistics, N50 value (the smallest contig size in which half the assembly is represented) [37, 38], the contig numbers of longer than $1000 \mathrm{bp}$ and the average contig size are always measured as a criterion in evaluating the performance of assemble and generated by common Perl scripts.

To non-model organisms, a sequence homology search, such as by BLASTX, against sequences from a wellannotated, phylogenetically related species is the most practical way to identify the quality of assemble result [39]. The breadth of genetic composition and the transcript contiguity were examined by leveraging a reference data set as accuracy and precision standard [40]. Since the genome of Trametes gallica, sweet potato and Oryza meyeriana were not available, 159, 312 and 532 identified protein sequences from them and their phylogenetically related species, Trametes versicolor laccase and Oryza sativa L, were chosen as reference databases. The megablast and the common Perl analysis script were used to analyze the representation.

In addition to the statistics of accuracy, another criterion, completeness, was used to evaluate the quality of the assemblies. Based on the blast results from accuracy evaluation, we considered the average of completeness of each assembly. Completeness, also known as integrity or transcriptome coverage, is the ratio of the sum of all unique aligned segment length to the reference length. We calculated the completeness with $\mathrm{Com}=\mathrm{TP} /(\mathrm{TP}+\mathrm{FN})$
( $\mathrm{TP}=$ true positives, $\mathrm{FN}=$ false negatives), where $\mathrm{Com}$ is completeness, TP is the sum of all aligned segment length (the overlap aligned regions were only calculated once), $\mathrm{FN}$ is the sum of all reference segment length that were not aligned.

Since most transcripts assembled from eukaryotic RNA-seq data derived from polyadenylated RNA are expected to code for proteins, the optimal assembly results will produce long and complete ORFs as many as possible. Potential coding regions within reconstructed transcripts were analyzed with the Perl script in the Trinity package. The open reading frames of size $900 \mathrm{bp}$ and $1200 \mathrm{bp}$ or longer were defined as long ORFs in this paper. The percentage of long ORF was compared among different de novo assemble tools and strategies.

\section{Availability of supporting data}

The full data sets of Trametes gallica have been submitted to NCBI Sequence Read Archive (SRA, http://www.ncbi. nlm.nih.gov/sra/) under Accession SRP050574, Bioproject: PRJNA263488. The full data sets of sweet potato and Oryza meyeriana have been submitted to NCBI SRA databases under Accession SRP050169 and SRP050359, Bioproject: PRJNA263487 and PRJNA263485.

\section{Additional files}

Additional file 1: Table S1. Comparison of de novo assembly strategies in Trametes gallica. 
Additional file 2: Table S2. Comparison of de novo assembly strategies in sweet potato.

Additional file 3: Table S3. Comparison of de novo assembly strategies in Oryza meyeriana.

Additional file 4: Table S4. Comparison of de novo assembly strategies in Zea mays.

Additional file 5: Figure S1. Comparsion of the length coverage of top database hits using various de novo assemblers in Zea mays and Triticum turgidum.

Additional file 6: Figure S2. Comparsion of number of long ORFs numbers and completeness using various de novo assemblers in Zea mays and Triticum turgidum. The left $\mathrm{Zm}$ and $\mathrm{Tr}$ indicates the number of size 900 bp or longer ORFs in Zea mays and Triticum turgidum.The right $\mathrm{Zm}$ and $\mathrm{Tr}$ indicates the performance of completeness in Zea mays and Triticum turgidum.

Additional file 7: Table S5. Comparison of de novo assembly strategies in Triticum turgidum.

\section{Abbreviations}

SASP: Single-Assembler Single-Parameter; SAMP: Single-Assembler Multiple-Parameters; CDTA: Combined De novo Transcriptome Assembly; OLC: Overlap-layout-consensus; ORF: Open Reading Frame.

\section{Competing interests}

The authors declare that they have no competing interests.

\section{Authors' contributions}

Conceived and designed the project: YZZ, BH. RNA isolation: $\mathrm{OHC}, \mathrm{CHW}$. Bioinformation analysis: BH, SRZ, YHC, XJC. Wrote the paper: BH, YZZ. All authors read and approved the final manuscript.

Received: 11 August 2014 Accepted: 22 December 2014

Published online: 08 February 2015

\section{References}

1. Logacheva MD, Kasianov AS, Vinogradov DV, Samigullin TH, Gelfand MS, Makeev VJ, et al. De novo sequencing and characterization of floral transcriptome in two species of buckwheat (Fagopyrum). BMC Genomics. 2011;12:30.

2. Hahn DA, Ragland GJ, Shoemaker DD, Denlinger DL. Gene discovery using massively parallel pyrosequencing to develop ESTs for the flesh fly Sarcophaga crassipalpis. BMC Genomics. 2009;10:234

3. Levin JZ, Berger MF, Adiconis X, Rogov P, Melnikov A, Fennell T, et al. Targeted next-generation sequencing of a cancer transcriptome enhances detection of sequence variants and novel fusion transcripts. Genome Biol. 2009:10:R115.

4. Montoya-Burgos Jl, Foulon A, Bahechar I. Transcriptome screen for fast evolving genes by Inter-Specific Selective Hybridization (ISSH). BMC Genomics. 2010;11:126.

5. Anders $\mathrm{S}$, Reyes A, Huber W. Detecting differential usage of exons from RNA-seq data. Genome Res. 2012;22:2008-17.

6. Johnson BR, Atallah J, Plachetzki DC. The importance of tissue specificity for RNA-seq: highlighting the errors of composite structure extractions. BMC Genomics. 2013;14:586.

7. Van Belleghem SM, Roelofs D, Van Houdt J, Hendrickx F. De novo transcriptome assembly and SNP discovery in the wing polymorphic salt marsh beetle Pogonus chalceus (Coleoptera, Carabidae). PLoS One. 2012;7:e42605

8. Ozsolak F, Milos PM. RNA sequencing: advances, challenges and opportunities. Nat Rev Genet. 2011:12:87-98

9. Robertson G, Schein J, Chiu R, Corbett R, Field M, Jackman SD, et al. De novo assembly and analysis of RNA-seq data. Nat Methods. 2010;7:909-12.

10. Warren RL, Sutton GG, Jones SJ, Holt RA. Assembling millions of short DNA sequences using SSAKE. Bioinformatics. 2007;23:500-1.

11. Hernandez $D$, Tewhey $R$, Veyrieras J-B, Farinelli $L$, Østerås $M$, François $P$, et al. De novo finished 2.8 Mbp Staphylococcus aureus genome assembly from 100 bp short and long range paired-end reads. Bioinformatics. 2014;30:40-9.
12. Schulz MH, Zerbino DR, Vingron M, Birney E. Oases: robust de novo RNA-seq assembly across the dynamic range of expression levels. Bioinformatics. 2012;28:1086-92.

13. Xie Y, Wu G, Tang J, Luo R, Patterson J, Liu S, et al. SOAPdenovo-Trans: De novo transcriptome assembly with short RNA-Seq reads. Bioinformatics. 2014;30:1643-50.

14. Ru L. SOAPdenovo2: an empirically improved memory-efficient short-read de novo assembler. Giga Sci. 2012;1:18.

15. Peng Y, Leung HC, Yiu S-M, Lv M-J, Zhu X-G, Chin FY. IDBA-tran: a more robust de novo de Bruijn graph assembler for transcriptomes with uneven expression levels. Bioinformatics. 2013;29:1326-34.

16. Haas BJ, Papanicolaou A, Yassour M, Grabherr M, Blood PD, Bowden J, et al. De novo transcript sequence reconstruction from RNA-seq using the Trinity platform for reference generation and analysis. Nat Protoc. 2013;8:1494-512.

17. Iorizzo M, Senalik DA, Grzebelus D, Bowman M, Cavagnaro PF, Matvienko M, et al. De novo assembly and characterization of the carrot transcriptome reveals novel genes, new markers, and genetic diversity. BMC Genomics. 2011;12:389

18. Nakasugi K, Crowhurst R, Bally J, Waterhouse P. Combining Transcriptome Assemblies from Multiple De Novo Assemblers in the Allo-Tetraploid Plant Nicotiana benthamiana. PLoS One. 2014;9:e91776.

19. Garg R, Patel RK, Tyagi AK, Jain M. De novo assembly of chickpea transcriptome using short reads for gene discovery and marker identification. DNA Res. 2011;18:53-63.

20. Zhang W, Chen J, Yang Y, Tang Y, Shang J, Shen B. A practical comparison of de novo genome assembly software tools for next-generation sequencing technologies. PLoS One. 2011;6:e17915.

21. Zhang J, Ruhlman TA, Mower JP, Jansen RK. Comparative analyses of two Geraniaceae transcriptomes using next-generation sequencing. BMC Plant Biol. 2013;13:228.

22. Kumar S, Blaxter ML. Comparing de novo assemblers for 454 transcriptome data. BMC Genomics. 2010;11:571.

23. Lin Y, Li J, Shen H, Zhang L, Papasian CJ, Deng HW. Comparative studies of de novo assembly tools for next-generation sequencing technologies. Bioinformatics. 2011;27:2031-7.

24. Chang Z, Wang Z, Li G. The Impacts of Read Length and Transcriptome Complexity for De Novo Assembly: A Simulation Study. PLoS One. 2014;9:e94825.

25. Tao X, Gu YH, Wang HY, Zheng W, Li X, Zhao CW, et al. Digital gene expression analysis based on integrated de novo transcriptome assembly of sweet potato [lpomoea batatas (L.) Lam]. PLoS One. 2012;7:e36234.

26. Tan L-Q, Wang L-Y, Wei K, Zhang C-C, Wu L-Y, Qi G-N, et al. Floral Transcriptome Sequencing for SSR Marker Development and Linkage Map Construction in the Tea Plant (Camellia sinensis). PLoS One. 2013;8:e81611.

27. Liang C, Liu X, Yiu S-M, Lim BL. De novo assembly and characterization of Camelina sativa transcriptome by paired-end sequencing. BMC Genomics. 2013;14:146

28. Krasileva KV, Buffalo V, Bailey P, Pearce S, Ayling S, Tabbita F, et al. Separating homeologs by phasing in the tetraploid wheat transcriptome. Genome Biol. 2013;14:R66.

29. Duan J, Xia C, Zhao G, Jia J, Kong X. Optimizing de novo common wheat transcriptome assembly using short-read RNA-Seq data. BMC Genomics. 2012;13:392.

30. Wang Z, Fang B, Chen J, Zhang X, Luo Z, Huang L, et al. De novo assembly and characterization of root transcriptome using Illumina paired-end sequencing and development of cSSR markers in sweetpotato (Ipomoea batatas). BMC Genomics. 2010;11:726.

31. Yang Y, Smith SA. Optimizing de novo assembly of short-read RNA-seq data for phylogenomics. BMC Genomics. 2013;14:328.

32. Cantu D, Pearce SP, Distelfeld A, Christiansen MW, Uauy C, Akhunov E, et al. Effect of the down-regulation of the high Grain Protein Content (GPC) genes on the wheat transcriptome during monocarpic senescence. BMC Genomics. 2011;12:492.

33. Schreiber AW, Hayden MJ, Forrest KL, Kong SL, Langridge P, Baumann U. Transcriptome-scale homoeolog-specific transcript assemblies of bread wheat. BMC Genomics. 2012;13:492.

34. Chow K-S, Ghazali A-K, Hoh C-C, Mohd-Zainuddin Z. RNA sequencing read depth requirement for optimal transcriptome coverage in Hevea brasiliensis. BMC Res Notes. 2014;7:69.

35. Schmieder R, Edwards R. Quality control and preprocessing of metagenomic datasets. Bioinformatics. 2011;27:863-4. 
36. Huang X, Madan A. CAP3: A DNA sequence assembly program. Genome Res. 1999;9:868-77.

37. Schliesky S, Gowik U, Weber AP, Bräutigam A. RNA-seq assembly-are we there yet? Frontiers Plant Sci. 2012;3:220.

38. Zeng V, Villanueva KE, Ewen-Campen BS, Alwes F, Browne WE, Extavour CG. De novo assembly and characterization of a maternal and developmental transcriptome for the emerging model crustacean Parhyale hawaiensis. BMC Genomics. 2011;12:581.

39. Steijger T, Abril JF, Engström PG, Kokocinski F, Hubbard TJ, Guigó R, et al. Assessment of transcript reconstruction methods for RNA-seq. Nat Methods. 2013;10:1177-84

40. Grabherr MG, Haas BJ, Yassour M, Levin JZ, Thompson DA, Amit I, et al. Full-length transcriptome assembly from RNA-Seq data without a reference genome. Nat Biotechnol. 2011;29:644-52.

\section{Submit your next manuscript to BioMed Central and take full advantage of:}

- Convenient online submission

- Thorough peer review

- No space constraints or color figure charges

- Immediate publication on acceptance

- Inclusion in PubMed, CAS, Scopus and Google Scholar

- Research which is freely available for redistribution 